\title{
Propagation Properties of an Astigmatic Cos- Gaussian Beam through Turbulent Atmosphere
}

\author{
Kaicheng Zhu ${ }^{1,3}$, Xiaolei $\mathrm{Ma}^{1}$, Chang Gao ${ }^{1}$, Dengjuan $\mathrm{Ren}^{1}$ and Jie Zhu ${ }^{2, *}$ \\ ${ }^{1}$ School of Engineering, Guangzhou College of Technology and Business, Guangzhou 510850, China \\ ${ }^{2}$ College of Science, Guizhou Institute of Technology, Guiyang 550003, China \\ ${ }^{3}$ School of Physics and Electronics, Central South University, Changsha 410083, China
}

\begin{abstract}
We use the extended Huygens-Fresnel integral to investigate the propagation properties of a cos-Gaussian beam (cosGB) with astigmatism in atmospheric turbulence. The intensity distribution behaviour along the propagation distance for an astigmatic cosGB in atmospheric turbulence are analytically and numerically demonstrated. Some novel phenomena are presented graphically, indicating that the intensity distribution and the on-axial intensity closely depend on the astigmatic parameter and the turbulent structure constant of the cosGBs in the atmospheric turbulence.
\end{abstract}

\section{Introduction}

Propagation of laser lights in a variety of media has garnered immense attention because of their applications in the wide range areas, such as optical communications, imaging and remote sensing [1]. Recent research has promoted an interesting laser beam named the Hermite-sinusoidal-Gaussian beam which are eigenmode of the paraxial wave equation. Extensive kinds of beam models such as sinh-(or cosh-) Gaussian and sin-(or cos-) Gaussian types belong to this specific family $[2,3]$. Their propagation behaviours through different optical systems or nonlocal nonlinear media have been well explored [4-8]. Statistical characteristics of these beams propagating in atmospheric turbulence are also important topics that has been attracting a great deal of theoretical and practical interest for a long time [9-16]. For instance, the light transmittance properties of the Hermite-coshGaussian and the Hermite-cosine-Gaussian beams in atmospheric turbulence have been investigated [10]. The spreading and the average intensity for the cosh-Gaussian beams travelling through atmospheric turbulence have been discussed in detail $[12,15,16]$. The relationship of atmospheric turbulence and the beam quality of cosine-Gaussian beams have been analysed [9]. Theoretical models for describing the reciprocity of the cosh-Gaussian and cosine-Gaussian beams in atmospheric turbulence have been developed [13]. Besides, for the cosine-Gaussian and cosh-Gaussian beams, the dependence of the scintillation index on the properties of the turbulent atmosphere have been demonstrated [11]. However, note that most of the aforesaid studies belong to this subject are limited to the no aberration case, which may not be sufficient for meeting the practical technological requirements.

*Corresponding author: jiezh_16@163.com 
Meanwhile, it is known that aberrations are a common event not only in an imperfect optical system but also in a laser diode like astigmatism. There is considerable research activity directed toward the dependence of the propagation properties on the astigmatic aberration of laser beams travelling through $\mathrm{ABCD}$ optical system or atmospheric turbulence [8, 14-16]. It has been reported that when a dark hollow beam with suitable astigmatism only exhibits a small beam waist size, its scintillation index value can be smaller than that without astigmatism especially in the far field $[13,16]$.

In this paper, we extend these discussions to explore the propagation behaviours of cosGBs in atmospheric turbulence taking the cross phase into consideration. According to the paraxial form of the extended Huygens-Fresnel principle, we derive an analytical model for such a beam with astigmatism travelling in the atmospheric turbulence. Furthermore, the intensity distribution and the spreading behaviour of such beams are demonstrated numerically. While the comparisons of the average intensity distribution between astigmatic and stigmatic cosGBs are discussed, some interesting and useful results are summarized at the end.

\section{Propagation of an astigmatic CosGB in atmospheric turbulence}

We use the Cartesian coordinate system in our calculation in which the $z$-axis is specified as the propagation axis. Assume the astigmatic $\cos G B$ in the source plane $z=0$ to be the following form $[8,16]$

$$
E\left(x_{0}, y_{0}\right)=E_{0} \exp \left(-\frac{x_{0}^{2}+y_{0}^{2}}{w_{0}^{2}}\right) \cos \left(\frac{a x_{0}}{w_{0}}\right) \cos \left(\frac{b y_{0}}{w_{0}}\right)
$$

where $w_{0}$ is the waist width of the Gaussian part, $a$ and $b$ are the coefficients of the hyperbolic sinusoidal function. Note that we focus on the astigmatism case where $a$ and $b$ are not equal in the following. Besides, let $E_{0}^{-2}=\pi w_{0}^{2}\left(1+e^{-a^{2} / 2}\right)\left(1+e^{-b^{2} / 2}\right) / 8$ to make the total power of the beam to be one unit.

Then, we use the extended Huygens-Fresnel integral to simulate the propagation of a cosGB with astigmatism in atmospheric turbulence:

$$
E(x, y, z)=\frac{-i}{\lambda z} \int d x_{0} d y_{0} E_{0}\left(x_{0}, y_{0}, 0\right) \exp \left\{-\frac{i k}{2 z}\left[\left(x-x_{0}\right)^{2}+\left(y-y_{0}\right)^{2}\right]+\psi\left(x_{0}, y_{0}, x, y\right)\right\}
$$

where $k=2 \quad$ with being the wavelength, and $\psi\left(x_{0}, y_{0}, x, y\right)$ is the solution to the Rytov method representing the random component of the complex phase for a spherical wave radiating from the incident plane to the receiver plane. The relationship of the average intensity of the astigmatic cosGB and the propagation distance $z$ in the atmospheric turbulence can be written as: 


$$
\begin{aligned}
I(x, y, z)= & \frac{1}{\lambda^{2} z^{2}} \int d x_{1} d y_{1} d x_{2} d y_{2}\left\langle E\left(x_{1}, y_{1}, z\right) E^{*}\left(x_{2}, y_{2}, z\right)\right\rangle \\
& \exp \left\{\frac{i k}{2 z}\left[\left(x-x_{1}\right)^{2}+\left(y-y_{1}\right)^{2}-\left(x-x_{2}\right)^{2}-\left(y-y_{2}\right)^{2}\right]\right\} \\
& \left\langle\exp \left[\psi^{*}\left(x_{1}, y_{1}, x, y\right)+\psi\left(x_{2}, y_{2}, x, y\right)\right]\right\rangle_{m}
\end{aligned}
$$

where the angle bracket implies the ensemble average of the medium statistics including the phase fluctuations and the log-amplitude induced by the atmospheric turbulence. According to the previous studies $[10,11]$, this ensemble average structure function can be described as

$$
\left\langle\exp \left[\psi^{*}\left(x_{1}, y_{1}, x, y\right)+\psi\left(x_{2}, y_{2}, x, y\right)\right]\right\rangle_{m}=\exp \left[-\frac{\left(x_{1}-x_{2}\right)^{2}+\left(y_{1}-y_{2}\right)^{2}}{\rho_{0}^{2}}\right]
$$

where $\rho_{0}=\left(0.545 C_{n}^{2} k^{2} z\right)^{-3 / 5}$ is the coherence length related to a spherical wave in atmospheric turbulence with $C_{n}^{2}$ being the refractive index structure constant whose value indicates the turbulent strength.

Substituting Eqs. (2)-(4) into Eq. (3) and introducing the following "sum" and "difference" coordinates

$$
q_{s}=\frac{q_{1}+q_{2}}{2 w_{0}} \quad(q=x, y) \quad q_{d}=\frac{q_{1}-q_{2}}{w_{0}} \quad(q=x, y)
$$

we obtain the following intensity distribution function

$$
\begin{aligned}
I(x, y, z) & =\frac{E_{0}^{2} w_{0}^{4}}{4 \lambda^{2} z^{2}} \int d x_{s} d y_{s} d x_{d} d y_{d}\left(e^{i a x_{d}}+e^{-i a x_{d}}+e^{2 i a x_{s}}+e^{-2 i a x_{s}}\right)\left(e^{i b y_{d}}+e^{-i b y_{d}}+e^{2 i b y_{s}}+e^{-2 i b y_{s}}\right) \\
& \exp \left[-2 x_{s}^{2}-2 y_{s}^{2}-\Omega\left(x_{d}^{2}+y_{d}^{2}\right)+2 i Z\left(x_{s} x_{d}+y_{s} y_{d}-x_{w} x_{d}-y_{w} y_{d}\right)\right] \\
& =\frac{E_{0}^{2} Z^{2}}{2 \pi^{2}}\left(I_{d x}+I_{s x}\right)\left(I_{d y}+I_{s y}\right)
\end{aligned}
$$

where $\Omega=\frac{w_{0}^{2}}{\rho_{0}^{2}}+\frac{1}{2}, Z=\frac{z_{R}}{z}, z_{R}=\frac{k w_{0}^{2}}{2}=\frac{\pi w_{0}^{2}}{\lambda}$ is the Rayleigh distance and $q_{w}=q / w_{0}$ $(q=x, y)$ are the scaled coordinates on the receiver plane. After lengthy but straightforward mathematical treatments, we arrives

$$
\begin{aligned}
& I_{d x}=\frac{\pi}{\sqrt{2 \Omega+Z^{2}}} \exp \left(-\frac{Z^{2} x_{w}^{2}+a^{2} / 4}{\Omega+Z^{2} / 2}\right) \cosh \left(\frac{a Z x_{w}}{\Omega+Z^{2} / 2}\right) \\
& I_{s x}=\frac{\pi}{\sqrt{2 \Omega+Z^{2}}} \exp \left(-\frac{Z^{2} x_{w}^{2}+\Omega a^{2} / 2}{\Omega+Z^{2} / 2}\right) \cos \left(\frac{a Z^{2} x_{w}}{\Omega+Z^{2} / 2}\right)
\end{aligned}
$$




$$
\begin{gathered}
I_{d y}=\frac{\pi}{\sqrt{2 \Omega+Z^{2}}} \exp \left(-\frac{Z^{2} y_{w}^{2}+b^{2} / 4}{\Omega+Z^{2} / 2}\right) \cosh \left(\frac{b Z y_{w}}{\Omega+Z^{2} / 2}\right) \\
I_{s y}=\frac{\pi}{\sqrt{2 \Omega+Z^{2}}} \exp \left(-\frac{Z^{2} y_{w}^{2}+\Omega b^{2} / 2}{\Omega+Z^{2} / 2}\right) \cos \left(\frac{b Z^{2} y_{w}}{\Omega+Z^{2} / 2}\right)
\end{gathered}
$$

Based on the above expressions, the propagation properties of a cosGB with or without astigmatism (separately featured by $a=b$ or $a \neq b$ ) in free space or in atmospheric turbulence (respectively corresponding to $C_{n}^{2}=0$ or $C_{n}^{2} \neq 0$ ) can be investigated in a convenient way.

\section{Numerical results and analysis}

We now proceed to analyse the evolution of the average intensity of an astigmatic or stigmatic cosGB propagating in atmospheric turbulence numerically and comparatively by setting the beam width as $800 \mathrm{~nm}, w_{0}=50 \mathrm{~mm}$ and the corresponding Rayleigh distance $z_{R} \approx 9.82 \mathrm{~km}$.

To understand the relationship between the spectral density behaviour and the stigmatic properties of the cosGBs propagating in atmospheric turbulence, Fig. 1 illustrates the intensity distribution evolutions at some selected distances for different values of the parameters $a$ and $b$. For the stigmatic case of $\mathrm{a}=\mathrm{b}=4$ as shown in Fig. 1(a), the intensity distribution $I(x, y, z)$ is a many-petal profile in the near field and quickly transforms to a Gaussian-like configuration in the far field. Meanwhile, for the astigmatic case of $a=1$ and $b=7$ as shown in Fig. 1(c), the intensity pattern which is still a many-petal profile in the near field, gradually fuses into a long ellipse spot shape and finally changes into a Gaussian-like intensity distribution after travelling a sufficiently long distance. In fact, performing calculations indicate that, as long as the astigmatism exist in which $a \neq b$, the intensity profile of an astigmatic cosGB always exhibits similar evolution behaviour aforesaid during the propagation in atmospheric turbulence. Moreover, by way of comparison the intensity evolutions of the weak astigmatic cosGB (with $a=3$ and $b=5$ ) propagating in atmospheric turbulence are shown in Fig.1(b).
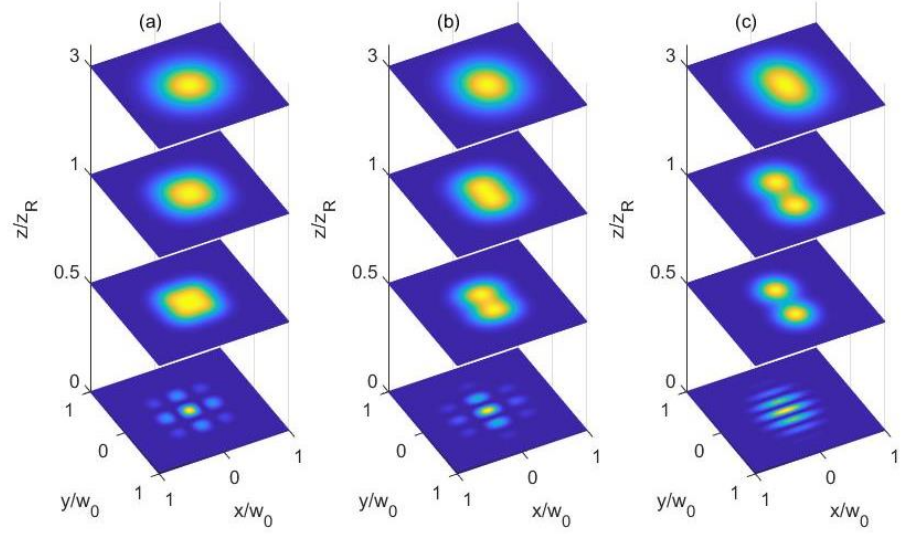

Fig. 1. On the different receiving planes average intensity distributions of CosGB without or with astigmatism propagating through atmosphere turbulence with strength coefficient $C_{n}^{2}=3 \times 10^{-16} \mathrm{~m}^{-2 / 3}$, where (a) $a=b=4$; (b) $a=3, b=5$ and (c) $a=1, b=7$. 
Next, let us consider the relationship between the on-axis intensity of cosGBs and the propagating distance in the atmospheric turbulence. It has been reported that when a stigmatic or astigmatic cosGB travels in free space, its on-axial intensity is always zero during propagation. Figure 2 illustrates the dependence of the on-axial intensity of the astigmatic cosGBs on the propagation distance $z$ in atmospheric turbulence. Obviously, the on-axial intensity monotonically decreases with increasing propagating distance, which is completely different from that in free space. Moreover, we point that the drop rate of the on-axial intensity depends markedly on the astigmatism of the beam and the atmosphere turbulence strength, which can be seen by comparison of Figs. 2(a) and 2(b).
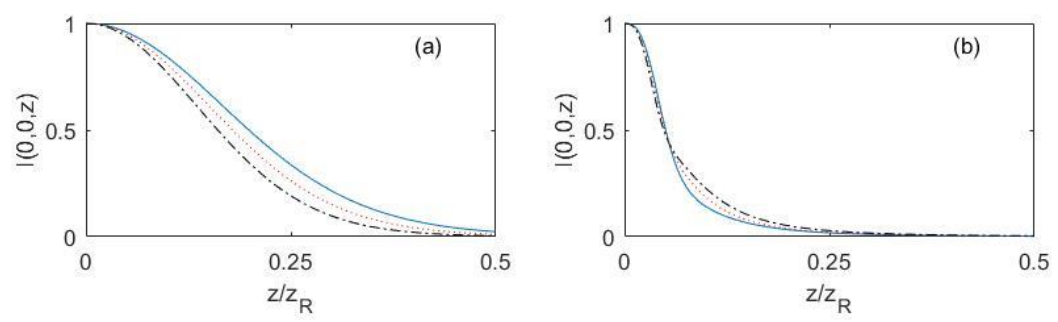

Fig. 2. On-axial intensity evolutions of $\mathrm{Cos} G B$ without or with astigmatism propagating through atmosphere turbulence. (a) $C_{n}^{2}=1 \times 10^{-16} \mathrm{~m}^{-2 / 3}$, solid line corresponding to $a=b=4$, dashed line to $a=2, b=6$ and dash-dotted line to $a=1, b=7$. (b) Same as (a) but $C_{n}^{2}=1 \times 10^{-14} m^{-2 / 3}$.

\section{Conclusions}

In conclusion, we have investigated how the astigmatism influences the intensity distribution of the cosGBs travelling through atmospheric turbulence. We have derived the analytical expressions of the average intensity of astigmatic cosGBs propagating in atmospheric turbulence, and numerically analysed the evolution of the spectral density for such a beam. It is shown that when the parameters sin-part parameter input beam width $w_{0}$ and astigmatic parameters $a$ and $b$ are suitable values, the average intensity pattern of an astigmatic cosGB experiences great variation during propagation. It is also proved that the structure constant $C_{n}^{2}$ and astigmatic parameter can effectively influence the variation speed of the intensity patterns. In a word, the results obtained in the paper open new perspectives for their potential applications, e.g., in laser illumination and laser communication through free space or atmospheric turbulence.

\section{References}

[1] Korotkova O 2014 Random Light Beams Theory and Applications (CRC Press)

[2] Casperson L W, Hall D G and Tovar A A 1997 J. Opt. Soc. Am. A 14 3341-3348

[3] Casperson L W and Tovar A A 1998 J. Opt. Soc. Am. A $15954-961$

[4] Zhao D, Mao H, Zhang W and Wang S 2003 Opt. Commun. 224 5-12

[5] Zhao D, Mao H, Liu H, Wang S, Jing F and Wei X 2004 Opt. Commun. 236 225-235

[6] Tang H Q and Zhu K C 2013 Opt. \& Laser Technology 3 68-71

[7] Zhu K C, Tang H Q, Tang Y and Xia H 2014 Opt. \& Laser Technol. 64 11-16 
[8] Shen S, Yang Z J, Guo J L, Wang Y J and Pang Z G 2020 Optik 224165454

[9] Eyyuboğlu H T 2005 Opt. Commun. 245 37-47

[10] Eyyuboğlu H T and Baykal Y 2007 Opt. Commun. 278 17-22

[11] Eyyuboğlu H T and Baykal Y 2007 Appl. Opt. 46 1099-1106

[12] Yang A L, Zhang E T, Ji X L and Lü B D 2008 Opt. Express 16, 8366-8380

[13] Eyyuboğlu H T and Baykal Y 2004 Opt. Express 12 4659-4674

[14] Chu X, Ni Y and Zhou G 2007 Appl. Phys. B 87 547-552

[15] Chu X 2007 Opt. Express 15 17613-17618

[16] Zhu J, Zhu K C, Tang H Q and Xia H 2017 Journal of Modern Optics 64 1915-1921 